\title{
OPEN B-box transcription factor 28 regulates flowering by interacting with constans
}

\begin{abstract}
Yin Liư ${ }^{1,2}$, Guang Lin $^{2}$, ChunmeiYin¹ \& Yuda Fang ${ }^{1,2}$
B-box transcription factors (BBXs) are important regulators of flowering, photomorphogenesis, shade-avoidance, abiotic and biotic stresses and plant hormonal pathways. In Arabidopsis, 32 BBX proteins have been identified and classified into five groups based on their structural domains. Little is known about the fifth group members (BBX26-BBX32) and the detailed molecular mechanisms relevant to their functions. Here we identified B-box transcription factor 28 (BBX28) that interacts with Constans (CO), a transcriptional activator of Flowering Locus $T$ (FT). Overexpressing BBX28 leads to late flowering with dramatically decreased $F T$ transcription, and $b b \times 28$ deficient mutant displays a weak early flowering phenotype under long days (LD), indicating that BBX28 plays a negative and redundant role in flowering under LD. Additionally, the interaction between BBX28 and CO decreases the recruitment of $\mathrm{CO}$ to $F T$ locus without affecting the transcriptional activation activity of $\mathrm{CO}$. Moreover, the $\mathrm{N}$-terminal cysteines, especially those within the B-box domain, are indispensable for the heterodimerization between $\mathrm{BBX} 28$ and $\mathrm{CO}$ and activation of $\mathrm{CO}$ on FT transcription. Genetic evidences show that the later flowering caused by $B B X 28$ overexpression is compromised by $C O$ ectopic expression. Collectively, these results supported that BBX28 functions with $C O$ and $F T$ to negatively regulate Arabidopsis flowering, in which the $\mathrm{N}$-terminal conserved cysteines of BBX28 might play a central role.
\end{abstract}

Flowering is an essential biological process for the transition from vegetative to reproductive stage in responses to internal and external signals ${ }^{1}$. Five main pathways have been identified in flowering regulation, including vernalization, autonomous, photoperiodic, gibberellin and age pathways ${ }^{2}$. Ambient temperature, sugar, histone modifications, small RNAs and chromatin loops also play roles in flowering regulation ${ }^{2,3}$. Constans (CO)-Flowering Locus $T(F T)$ is the central module in photoperiodic pathway ${ }^{4}$. CO, the first member of B-box transcription factors (BBX1), binds to the CORE1 and CORE2 motifs of FT promoter to activate its expression ${ }^{5}$. FT interacts with bZIP transcription factor FD to promote the expressions of floral identity genes and induce floral meristem formation ${ }^{6}$.

The transcriptional and post-translational regulations of $\mathrm{CO}$ ensure the precise protein level of $\mathrm{CO}$ during the day-night and the correct time of FT expression under long day (LD) condition to induce photoperiodic flowering ${ }^{4,7}$. At the post-translational level, the stability and activity of CO protein are regulated by multiple CO-interacting proteins ${ }^{4,7}$. Two RING-finger E3 ubiquitin ligases Constitutive Photomorphogenic 1 (COP1) and High Expression of Osmotically Responsive Genes 1 (HOS1) mediate the degradation of CO at night and in the morning respectively ${ }^{8,9}$. Photoreceptors Phytochrome B (PHYB) and Zeitlupe (ZTL) destabilize CO in the morning while Phytochrome A (PHYA), Cryptochromes (CRYs) and Flavin-Binding, Kelch Repeat F-box1 (FKF1) stabilize CO in the afternoon under $\mathrm{LD}^{10-13}$. The interaction between Nucleoporin 96 (Nup 96) and HOS1 and their mutual stabilization form a novel repressive module to gate CO protein level in Arabidopsis under $\mathrm{LD}^{14}$. GIGANTEA (GI) plays a pivotal regulation on the timing stabilization of CO by altering FKF1ZTL interaction ${ }^{15}$. Other CO-interacting proteins, including BBX19, microProtein 1a (miP1a, or BBX30) and miP1b (or BBX31), Target of EAT1 (TOE1), TOE2, Nuclear Factor Y (NF-Y) transcription factors, Della protein RGA, Botrytis Susceptible 1 Interactor (BOI), Vascular Plant One-Zinc Finger 1 (VOZ1), VOZ2 and immunophilin FKBP12, regulate flowering by affecting CO transcriptional activity or DNA binding ability ${ }^{16-23}$. Pseudo Response Regulator (PRR) proteins regulate both $\mathrm{CO}$ transcription and $\mathrm{CO}$ protein stabilization, increasing the recruitment of CO to FT promoter ${ }^{24}$. CRY2-interacting bHLH1(CIB1), CO and CRY2 form a complex through

\footnotetext{
${ }^{1}$ Joint Center for Single Cell Biology, School of Agriculture and Biology, Shanghai Jiao Tong University, Shanghai 200240, China. ${ }^{2}$ National Key Laboratory of Plant Molecular Genetics, CAS Center for Excellence in Molecular Plant Sciences, Institute of Plant Physiology and Ecology, Chinese Academy of Sciences, University of Chinese Academy of Sciences, Shanghai 200032, China. ${ }^{\varpi}$ email: yuda.fang@sjtu.edu.cn
} 
physical interaction between CIB1 and CO to regulate FT expression and CRY2-dependent flowering ${ }^{25}$. Long Hypocotyl in FAR-RED 1 (HFR1) interacts with CO and Phytochrome-Interacting Factor 7 (PIF7) to repress early flowering under the shaded environment ${ }^{26}$.

B-box transcription factors are a class of zinc finger binding proteins, which contain one or two B-box domains in the N-terminus and sometimes a CCT (CONTANS, CO-like and TOC1) domain in the C-terminus $^{27,28}$. Based on the numbers of B-box domains and the existence of CCT domain, the 32 B-box proteins in Arabidopsis are classified into five groups. BBX26-BBX32 belong to the fifth group, which contains only one B-box domain in the $\mathrm{N}$-terminus but no CCT domain in the $\mathrm{C}$-terminus ${ }^{27}$. BBX1 (CO) and many other B-box proteins were known to regulate flowering in CO-dependent or independent manners ${ }^{27}$. The suppression of flowering by BBX4 (COL3) depends on the BBX32-BBX4 interaction which contributes to the targeting of $\mathrm{BBX} 4$ to $F T$ promoter to inhibit $F T$ expression ${ }^{29,30}$. Overexpressing BBX6 causes early flowering by increasing the transcripts of FT and SUPPRESSOR OF OVEREXPRESSION OF CO 1 (SOC1) but not CO under short day (SD) condition ${ }^{31}$. Overexpression of BBX7 delays flowering under LD through decreasing CO, FT and SOC1 mRNA levels ${ }^{32}$. BBX19 decreases FT transcript level and represses flowering under LD $^{16}$. BBX24 (SALT TOLERANCE, STO) promotes flowering under both SD and LD conditions by reducing FLOWERING LOCUS C (FLC), FT and SOC1 expressions ${ }^{33}$. BBX30 and BBX31 interact with CO and TOPLESS (TPL) to suppress FT expression $^{19}$. BBX32 interacts with EMBRYONIC FLOWER 1 (EMF1) to regulate flowering ${ }^{34}$. Heading date 1(Hd1), OsBBX5, OsBBX14, OsBBX27 or OsCOL9 in rice play positive or negative roles in flowering under LD or SD conditions ${ }^{35-39}$. Chrysanthemum BBX8 and BBX13 were reported to regulate flowering ${ }^{40,41}$. At the cellular level, $\mathrm{CO}$ and BBX4 colocalize with Constitutive Photomorphogenic 1 (COP1) in photobodies through interaction with COP $1^{9,30}$.

In this study, we identified a new CO-interacting protein BBX28 and revealed that BBX28 functions through its $\mathrm{N}$-terminal cysteine-mediated heterodimerization with $\mathrm{CO}$ to weaken the role of $\mathrm{CO}$ in transcriptional regulation of FT to negatively regulate Arabidopsis flowering.

\section{Results}

BBX28 negatively regulates flowering. To uncover the biological functions of BBX28 in addition to its role in the regulation of photomorphogenesis ${ }^{42}$, we obtained a $b b \times 28$ mutant with a T-DNA insertion in the first exon without a detectable BBX28 transcript level (Fig. S1a,b), and two independent transgenic lines overexpressing $B B X 28$ fused to yellow fluorescent protein (YFP) under the control of a CaMV 35S promoter in wild type (Col-0) (BBX28OE, 35S-BBX28-YFP/Col-0; lines 1\# and 4\#) (Fig. S1b), while Col-0 and plants overexpressing YFP (YFPOE, 35S-YFP/Col-0) were used as controls. When these plants were grown under LD, bbx28 mutant flowered two days earlier with one rosette leaf less than Col-0, while BBX28OE lines showed severely late flowering phenotype, with about twenty days later and ten more rosette leaves than Col-0 (Fig. 1a-c). As the two independent $B B X 28$-overexpressing lines display similar phenotypes (Fig. 1a-c), we used BBX28OE-1\# for further analysis.

We crossed $B B X 28 \mathrm{OE}$ with $b b \times 28$ mutant to obtain $B B X 28 \mathrm{OE} / b b \times 28$ plants. We found that $B B X 28 \mathrm{OE} / b b \times 28$ plants flowered similarly to $B B X 28 \mathrm{OE}$ plants (Fig. S1c-e). The weak phenotype of $b b \times 28$ mutant might be due to the functional redundancies of Arabidopsis group V BBX proteins in flowering regulation (Fig. S1f).

To map the functional domains of BBX28 in flowering repression, we constructed N-terminal (BBX28NT, 1-112 aa) and C-terminal (BBX28CT, 113-223 aa) truncations of BBX28 based on their protein domains predicted by SMART (https://smart.embl-heidelberg.de/), which revealed a B-box domain (1-46 aa) in the $\mathrm{N}$-terminus and two low-complexity regions in the C-terminus of BBX28 (Fig. S1g). We generated transgenic plants overexpressing BBX28NT or BBX28CT in Col-0 (BBX28NTOE, 35S-BBX28NT-YFP/Col-0; BBX28CTOE, $35 S-B B X 28 C T-Y F P / C o l-0)$ (Fig. S1h). We found that BBX28NTOE lines showed severely late flowering, with about twenty days later and ten more rosette leaves than Col-0. In contrast, BBX28CTOE and YFPOE lines displayed similar flowering phenotypes to Col-0 (Fig. 1d-f), suggesting a specific role of BBX28NT in the flowering regulation.

BBX28 decreases FT transcription. To reveal genes involved in the negative role of BBX28 in regulating flowering, we tested the transcript levels of key genes involved in photoperiodic flowering, floral identity and $F T$ transcription, including CO, FT, SOC1, LEAFY (LFY), FRUITFUL (FUL), TEMPRANILLO 1 (TEM1), FLC and SHOT VEGETATIVE PHASE $(S V P)^{16}$, in 7-day-old LD-grown Col-0, bbx28 and BBX28OE plants. Quantitative real-time PCR (qRT-PCR) of seedlings collected at zeitgeber time (ZT) 4 showed that $F T$ transcript level reduced most significantly in BBX28OE, followed by SOC1, LFY, FUL and SVP, while CO, TEM1 and FLC transcript levels remained unchanged (Fig. 2a).

As the expressions of $C O$ and FT show rhythmic patterns during $24 \mathrm{~h}$ of a day in flowering pathway ${ }^{4}$, we tested whether the diurnal expression profiles of $F T$ and $C O$ are affected by BBX28. To this end, 7-day-old Col-0, $b b \times 28$ and BBX28OE seedlings grown under LD were harvested with an interval of three hours from dawn up to a 24 h-period. We found that FT transcript level was greatly decreased by BBX28 with more dramatically in late afternoon and dark (Fig. 2b). Interestingly, $\mathrm{CO}$ transcription was not affected by BBX28 during the day but decreased about half in BBX28OE plants after dusk (Fig. 2c).

BBX28 interacts with CO through its $\mathbf{N}$-terminus. Since FT is the direct target of CO, the reduction of FT transcript level by BBX28 encouraged us to test whether BBX28 interacts with CO. Co-localization analyses revealed that BBX28 and BBX28NT but not BBX28CT co-localized with CO in photobodies (Fig. 3a). We performed NoTS assay ${ }^{43}$ to test the interaction between BBX28 and CO in vivo. We found that CO was successfully recruited to the periphery of nucleolus by Nuc2-BBX28 but not Nuc2-mCherry (Fig. 3b), indicating that BBX28 
a

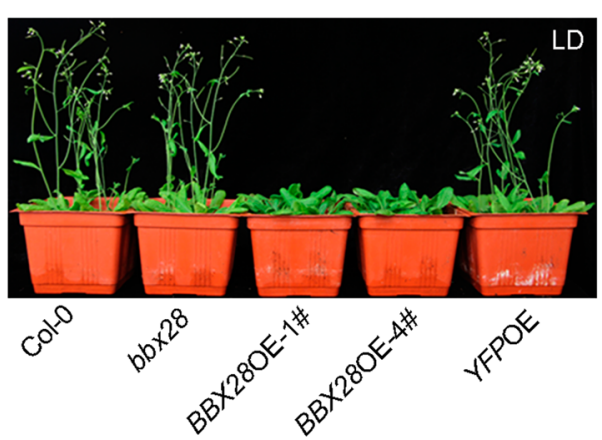

b

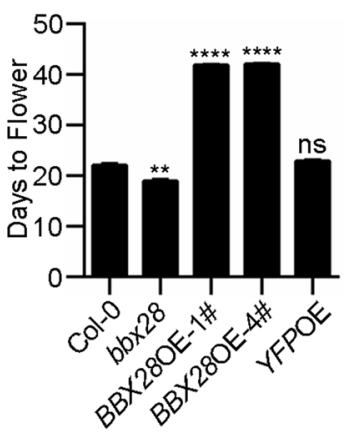

$c$

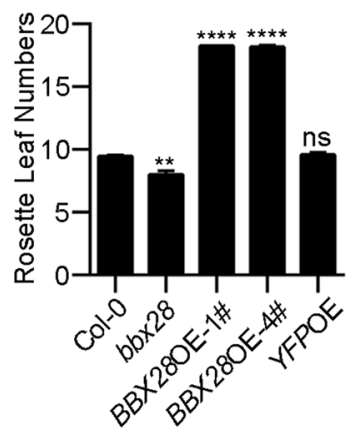

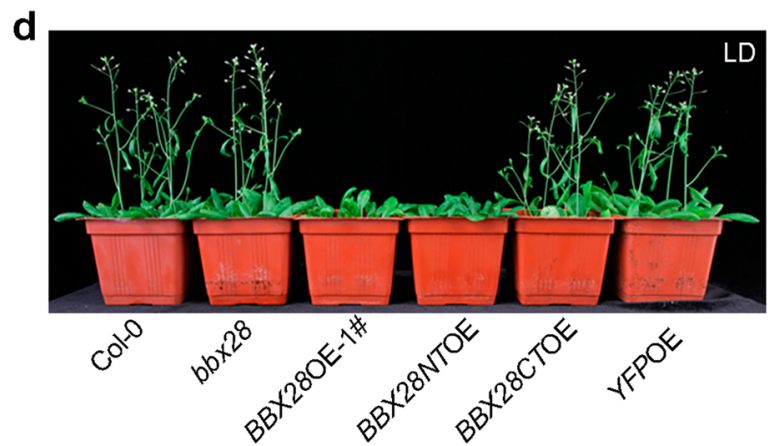

e

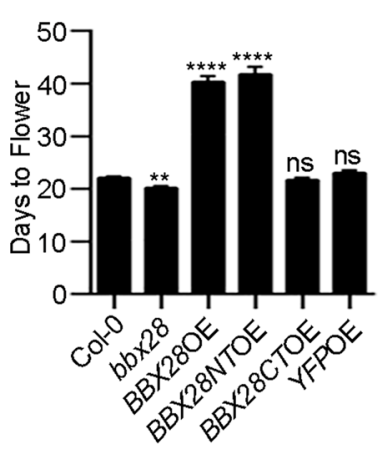

f

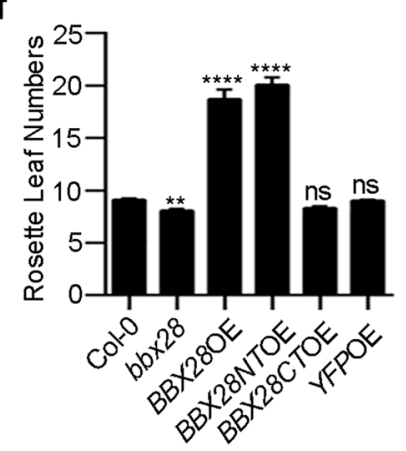

Figure 1. BBX28 negatively regulates flowering. (a) Visual phenotypes of 33-day-old Col-0, bbx28, and plants overexpressing YFP or BBX28 under LD. BBX28OE: 35S-BBX28-YFP/Col-0; YFPOE: 35S-YFP/Col-0. Numerals with the pound sign represent independent lines. (b, c) Flowering time and rosette leaf numbers of genotypes in (a) $(\mathrm{n}=4$ biological replicates; plant number $\geq 9$ in each replicate). (d) Visual phenotypes of 33-day-old Col-0, $b b \times 28$, and plants overexpressing $B B X 28$ or its truncations under LD. BBX28NTOE: $35 S-B B X 28 N T-Y F P / C o l-$ 0; BBX28CTOE: 35S-BBX28CT-YFP/Col-0. (e, f) Flowering time and rosette leaf numbers of genotypes in (d) $(\mathrm{n}=5$ biological replicates; plant number $\geq 9$ in each replicate). Data are means \pm SEM. Statistical significance was analyzed by student's $t$-test; ${ }^{* * *} p<0.0001,{ }^{* *} p<0.01$; ns, not significant.

interacts with $\mathrm{CO}$ in nuclei in vivo. LUC complementation imaging and yeast two-hybrid interaction assays also supported the interaction between BBX28 and CO through BBX28NT (Fig. 3c,d). The in vivo interaction between BBX28 and CO was further confirmed by Co-IP as CO was detected in BBX28-YFP-immunoprecipitated samples but not in the control (Figs. 3e; S2a). To study whether the B-box domain within N-terminus of BBX28 mediates its interaction with CO, BBX28 B-box (1-46aa) and BBX28NT with a deletion of the B-box domain (BBX28NT $\Delta$ B-box, 47-112aa) were constructed (Fig. S2b). Yeast two-hybrids showed that the B-box domain but not BBX28NT $\triangle \mathrm{B}$-box interacts with $\mathrm{CO}$ (Fig. S2c), suggesting a central role of B-box domain in mediating the interaction between BBX28 and CO.

BBX28 inhibits CO targeting to FT locus without affecting the transcriptional activation activity of $\mathrm{CO}$. To investigate the functional links among BBX28, CO and $F T$, we first compared the expression patterns among $B B X 28, C O$ and $F T$. Transgenic plants expressing GUS driven by the $B B X 28$ promoter in wild type (pBBX28-GUS/Col-0) were generated and GUS staining showed that the expression pattern of $B B X 28$ is similar to those of $C O$ and $F T^{44,45}$, with higher levels in vascular tissues (Fig. 4a). Dual-LUC assays (Fig. S3a) showed that the FT expression level was increased by CO but decreased by BBX28 (Fig. 4b), indicating a negative effect of BBX28 on FT transcription. Since CO activates FT transcription and BBX28 interacts with CO (Figs. 3 and 4b), we co-expressed BBX28 and CO and performed Dual-LUC assays to test the effect of coexpressing $B B X 28$ and $C O$ on $F T$ expression. The results indicated that the $F T$ transcript level was reduced upon co-expressing BBX28 and CO (Fig. 4b,c). Moreover, BBX28NT but not BBX28CT displayed a similar effect on $F T$ transcription to that of full-length BBX28 upon co-expressing with $\mathrm{CO}$ (Fig. 4c).

It was known that $\mathrm{CO}$ binds to FT promoter to activate its expression ${ }^{5,17}$. We asked whether BBX28 affects the DNA binding ability of $\mathrm{CO}$ to FT locus. Plants overexpressing CO (COOE; 35S-CO-YFP/Col-0) were crossed with $B B X 28 \mathrm{OE}$ line (BBX28OE; 35S-BBX28-3 $\times$ FLAG-mCherry/Col-0) to obtain CO and BBX28 co-expressing lines $(B B X 28 \mathrm{OE} \times C O O E)$. The line $1 \#, 3 \#$, and $5 \#$ of $B B X 28 \mathrm{OE} \times C O O E$ were generated by crossing $B B X 28 \mathrm{OE}-2 \#$ with line 1\#, 2\#, and 6\# of COOE respectively (Fig. S3b,c). COOE-2\# and BBX28OE $\times$ COOE-3\#, having a similar CO protein level which excluded the potential effects of different CO levels on our analysis (Fig. S3d), were selected for chromatin immunoprecipitation (ChIP)-qPCR assay. The level of CO recruited to the transcription start site of FT (FT2 fragment) was much more than that to other fragments of FT in COOE plants, exhibiting enrichment over four folds compared to the non-immune control (NIC) (Figs. 4d, S3e). When CO was co-expressed with BBX28, the level of CO targeted to FT2 fragment decreased to less than two folds compared to NIC (Fig. 4d), indicating that BBX28 decreases the recruitment of $\mathrm{CO}$ to FT locus. 
a
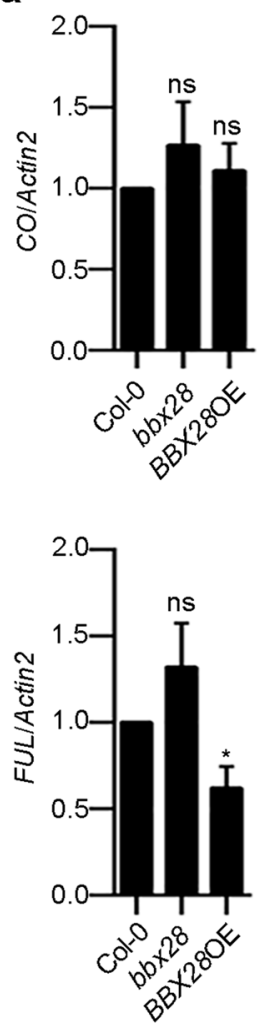
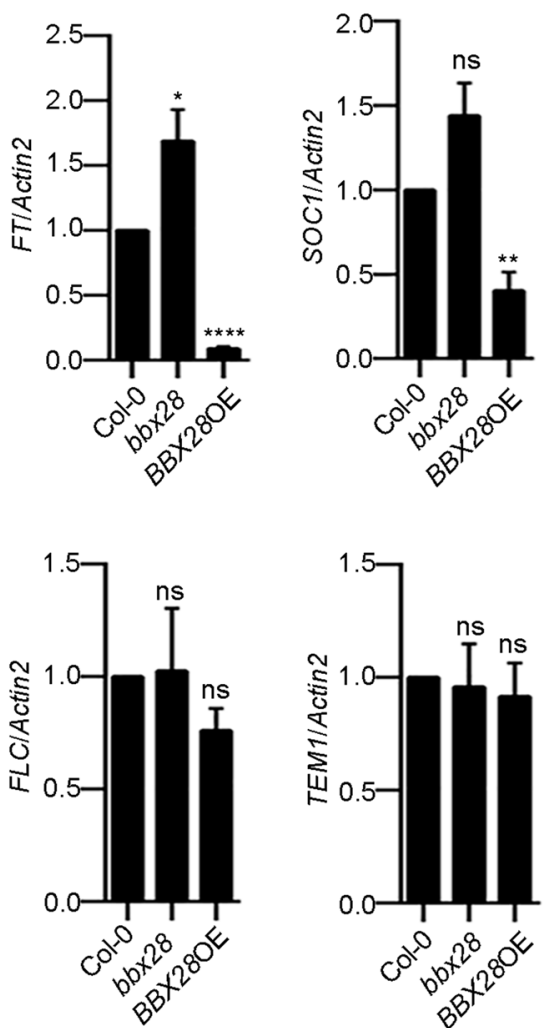
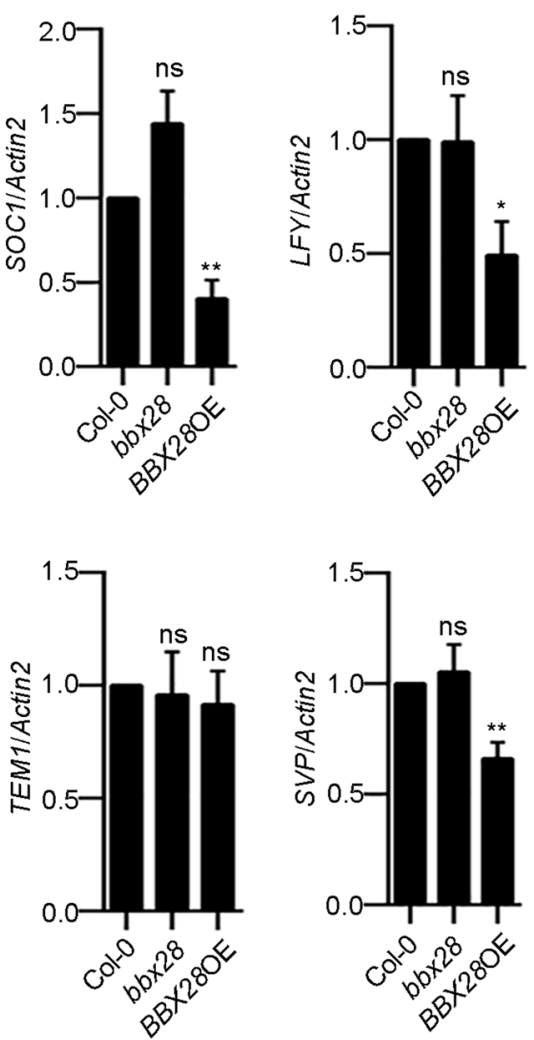

b

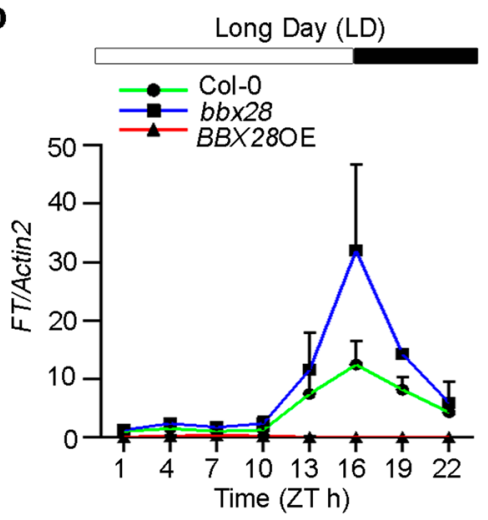

C

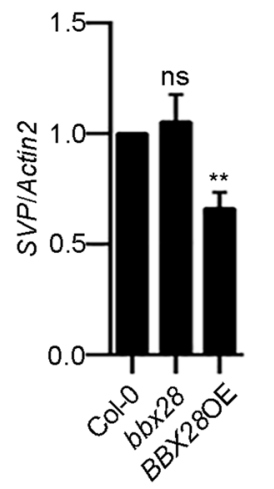

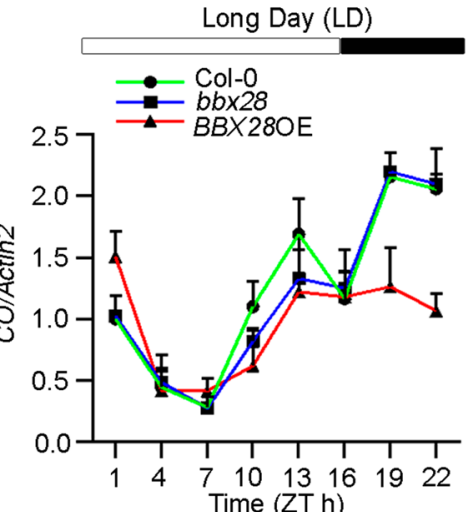

Figure 2. BBX28 decreases FT transcription. (a) Transcript levels of flowering-related genes in 7-day-old LD-grown Col-0, bbx28 and BBX28OE (35S-BBX28-YFP/Col-0) Arabidopsis plants. Seedlings were collected at zeitgeber time (ZT) 4 for RNA extraction and qRT-PCR assays ( $\mathrm{n}=6$ biological replicates). (b, c) Diurnal levels of FT transcript (b) or CO transcript (c) in Col-0, bbx28 and BBX28OE plants ( $\mathrm{n}=3$ biological replicates). Actin2 was used for data normalization in $\mathrm{qRT}$-PCR assays. Data are means \pm SEM. Statistical significance was analyzed by student's $t$-test; ${ }^{* * *} p<0.0001,{ }^{* *} p<0.01,{ }^{\star} p<0.05$; ns, not significant.

Next we tested whether BBX28 affects the transcriptional activation activity of CO (Fig. S3f). The expression of $L U C$ (LUC/REN ratio) increased in Gal4BD-CO-YFP and Gal4BD-VP16-YFP (Fig. S3g), consistent with the previous report ${ }^{5}$. When Gal4BD-CO-YFP was co-expressed with BBX28 or TOE1, a positive control which was shown to repress the transcriptional activation of $\mathrm{CO}^{18}$, we found that the transcriptional activation activity of CO was not affected by BBX28 while inhibited obviously by TOE1 (Fig. 4e). Without Gal4BD-CO-YFP, BBX28 and TOE1 had no effects on the reporter $L U C$ (Fig. S3h). Together, these results indicated that BBX28 does not affect the transcriptional activation activity of $\mathrm{CO}$.

BBX28 N-terminal cysteines mediate heterodimerization between BBX28 and CO and affect the activation of $\mathrm{CO}$ on $\boldsymbol{F T}$ transcription. As BBX28 interacts with $\mathrm{CO}$ through its N-terminus (Fig. 3), we then asked whether the conserved cysteines in N-terminal domain (Fig. S4a) play a role in BBX28-CO interaction. We found that mutations of BBX28 N-terminal cysteines greatly blocked BBX28-CO interaction with $\mathrm{BBX} 28^{\mathrm{C} 5,8 \mathrm{~A}}, \mathrm{BBX} 28^{\mathrm{C} 16 \mathrm{~A}}$ and $\mathrm{BBX} 28^{\mathrm{C} 24,27 \mathrm{~A}}$ displaying more obvious blocking effects, followed by $\mathrm{BBX} 28^{\mathrm{C} 47,50 \mathrm{~A}}$ and BBX28 ${ }^{\mathrm{C} 70,73 \mathrm{~A}}$ (Fig. 5a). Consistently, the co-localization signals in photobodies between $\mathrm{CO}$ and $\mathrm{BBX} 28^{\mathrm{C} 5,8 \mathrm{~A}}$ or $\mathrm{BBX} 28^{\mathrm{C} 16 \mathrm{~A}}$ decreased significantly, followed by $\mathrm{BBX} 28^{\mathrm{C} 24,27 \mathrm{~A}}, \mathrm{BBX} 28^{\mathrm{C} 47,50 \mathrm{~A}}$ and $\mathrm{BBX} 28^{\mathrm{C} 70,73 \mathrm{~A}}$ mutants. $\mathrm{BBX} 28^{\mathrm{C} 5-\mathrm{C} 73 \mathrm{~A}}$ with all nine conserved cysteines mutated diffused in the nucleus without photobodies formed when co-expressing with $\mathrm{CO}$ (Fig. S4b,c). These results indicated that the $\mathrm{N}$-terminal cysteines are essential for BBX28-CO interaction and C5, C8, C16, C24 and C27 sites located in the B-box domain (1-46aa) are more important. As BBX28 and CO (BBX1) both belong to BBX proteins, we concluded that BBX28 N-terminal cysteines mediated its heterodimerzation with $\mathrm{CO}$.

To test whether these cysteines affect $F T$ transcription, Dual-LUC assays showed that BBX28 cysteine mutations did not change the effects of BBX28 on FT expression, except BBX28 ${ }^{\mathrm{C} 5,8 \mathrm{~A}}$ mutation with less than one-fold increase (Fig. 5b). When those mutations were co-expressed with CO, BBX28 $8^{\mathrm{C} 5,8 \mathrm{~A}}, \mathrm{BBX} 28^{\mathrm{C} 16 \mathrm{~A}}, \mathrm{BBX} 28^{\mathrm{C} 24,27 \mathrm{~A}}$ and $\mathrm{BBX} 28^{\mathrm{C} 5-73 \mathrm{~A}}$ mutations, which affect the interaction between BBX28 and CO (Fig. 5a; Fig. S4b,c), largely restored FT expression (Fig. 5c), supporting that these cysteines play an inhibitory role in the activation of CO on FT transcription, possibly by affecting the heterodimerization between BBX28 and CO. 
a
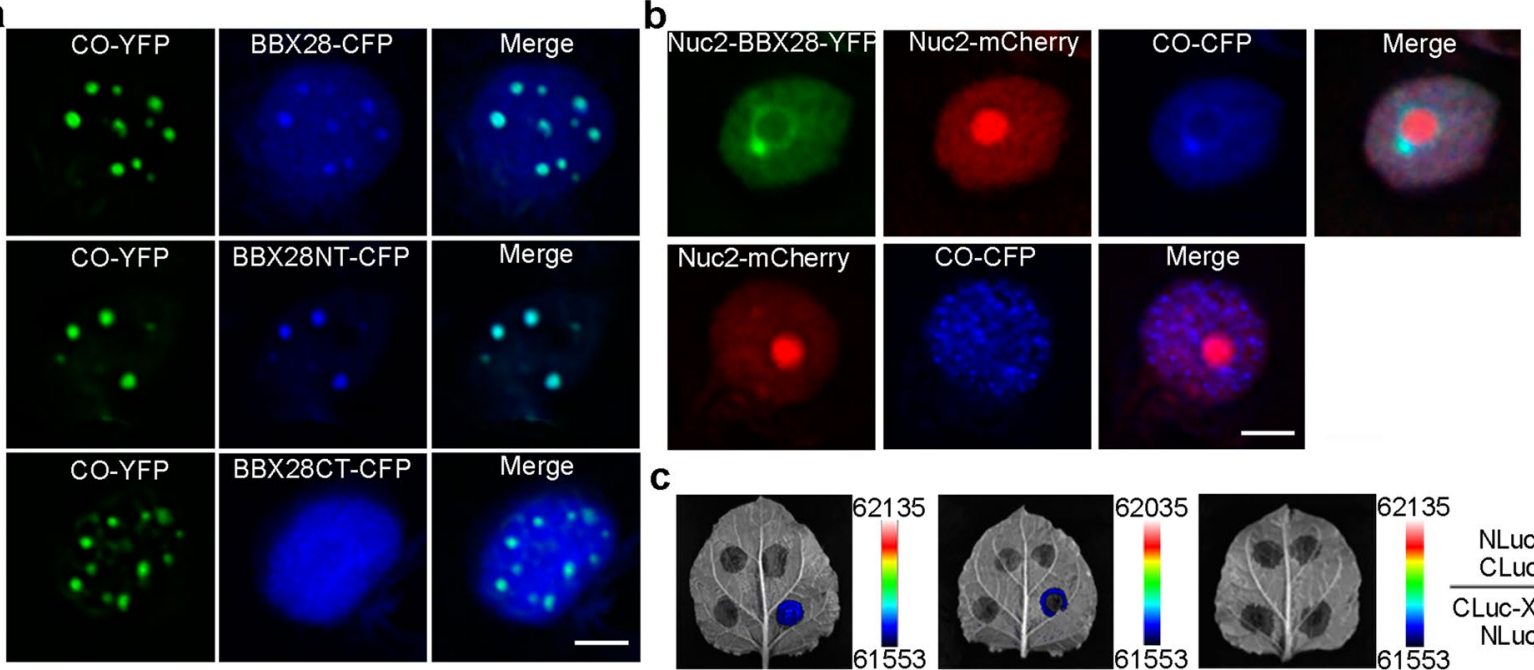

C
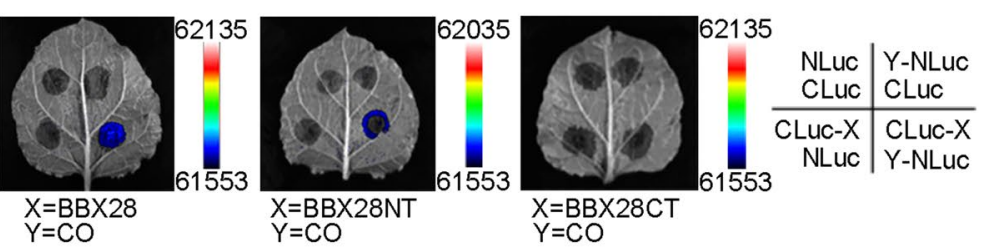

d

$\mathrm{BD}$

$\mathrm{BD}-\mathrm{BB} \times 28$

BD-BBX28NT $\mathrm{BD}-\mathrm{BB} 228 \mathrm{CT}$

$\mathrm{BD}$

$\mathrm{BD}-\mathrm{BB} \times 28$

$\mathrm{BD}-\mathrm{BB} \times 28 \mathrm{NT}$

BD-BBX28CT

$\mathrm{BD}$

$\mathrm{BD}-\mathrm{BB} \times 28$

$\mathrm{BD}-\mathrm{BB} \times 28 \mathrm{NT}$

$\mathrm{BD}-\mathrm{BB} \times 28 \mathrm{CT}$
AD $\quad A D-C O$

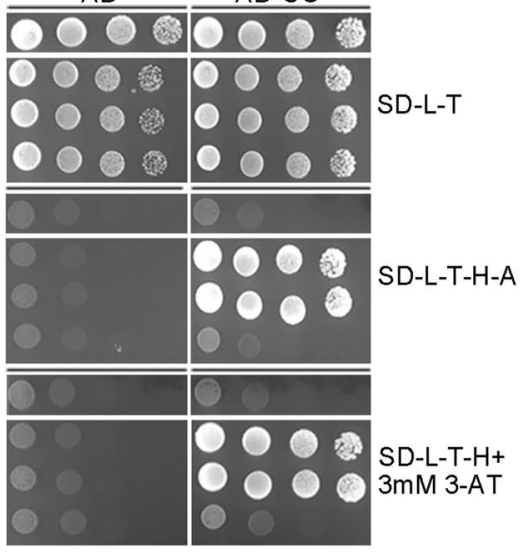

e

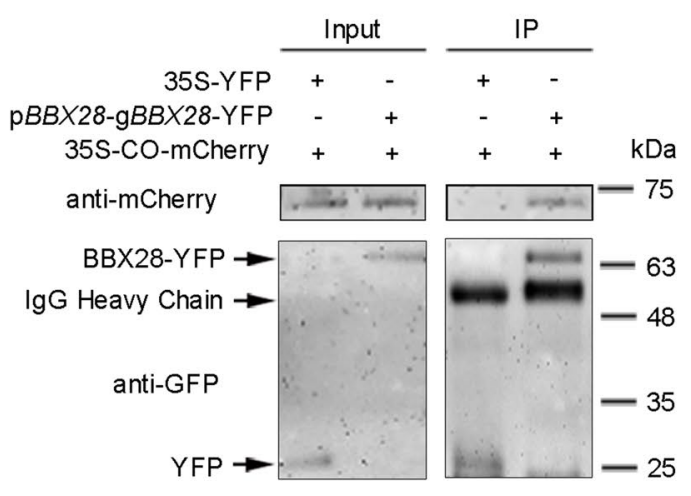

Figure 3. BBX28 interacts with CO through its N-terminus. (a) Co-localizations of CO and BBX28 in tabacco leaves. Bar $=5 \mu \mathrm{m}$. (b) NoTS assays of the interaction between BBX28 and CO in tobacco. The nucleolus was labeled by Nuc2-mCherry. Bar $=5 \mu \mathrm{m}$. (c) LUC complementation imaging assays of CO-BBX28 interaction in tobacco. NLuc/CLuc, Y-NLuc/CLuc and NLuc/CLuc-X acted as negative controls. (d) Yeast two-hybrids of BBX28 or BBX28 truncations with CO. Cells were grown on selective plates for interaction assays. (e) Co-IP assay of BBX28-CO interaction. Tobacco leaves co-expressing CO-mCherry and YFP were used as the negative controls. Proteins were detected by western blots with anti-GFP or anti-mCherry antibodies. Molecular weight standards are indicated. The full-length blots are presented in Fig. S2a.

BBX28 genetically regulates flowering through $\mathrm{CO}$ and $\boldsymbol{F T}$. To test the genetic relationships among $B B X 28$, $C O$ and $F T$, we first generated $b b \times 28 c o-9$ double mutant by crossing $b b \times 28$ with co- 9 mutant and analyzed their flowering phenotypes under LD. We found $b b \times 28 c o-9$ double mutant delayed flowering similarly to $c o-9$ single mutant (Fig. S5a-c). As the early flowering phenotype of $b b \times 28$ is weak (Fig. 1) while co mutant (co-9) displays severe late flowering (Fig. S5a-c), we then tested the genetic relationship between $B B X 28$ and $C O$ through analyzing the flowering phenotypes of plants overexpressing $B B X 28$ (BBX28OE), $C O$ (COOE) or $B B X 28$ and $C O(B B X 28 \mathrm{OE} \times C O O E)$. The line $1 \#, 3 \#$, and $5 \#$ of $B B X 28 \mathrm{OE} \times C O O E$ were generated by crossing $B B X 28 \mathrm{OE}-2 \#$ with line $1 \#, 2 \#$, and 6\# of COOE respectively, and the transcript levels of $C O$ and $B B X 28$ were confirmed by qRT-PCRs (Fig. S3b,c). We found that $B B X 28 \mathrm{OE} \times C O O E-5 \#$ flowered early with about twelve days earlier and five rosette leaves less than Col-0, similar to COOE-6\# plants (Fig. 6a-c); BBX28OE $\times$ COOE-1\# flowered early with about eleven days earlier and four rosette leaves less than Col- 0 , similar to COOE- $1 \#$ plants (Fig. S5d-f); BBX28OE $\times$ COOE-3\# flowered early with about ten days earlier and four rosette leaves less than Col-0, similar to COOE-2\# plants (Fig. S5g-i), indicating the later flowering caused by BBX28 overexpression was compromised by $C O$ overexpression. $B B X 28 \mathrm{OE} \times C O O E$ plants flowered no later than COOE plants, which might be due to that the high levels of $C O$ transcripts in both genotypes, resulting in a saturated level of $F T$ transcript for promoting flowering. Moreover, $F T$ transcripts were much less in $B B X 28 \mathrm{OE} \times C O O E$ plants than that in COOE plants (Fig. 6d), although the $C O$ transcript and $C O$ protein levels were comparable in $B B X 28 \mathrm{OE} \times C O O E$ plants and corresponding COOE plants (Fig. S3b,d), supporting a negative effect of BBX28 on CO function in vivo. In addition, $F T$ mRNA level changed in accordance with $C O$ transcript level in $B B X 28 \mathrm{OE} \times C O O E$ plants (1\#, 3\# and 5\#) (Figs. 6d, S3b). BBX28OE $\times$ COOE-3\# showed the highest CO transcript level, followed 
a

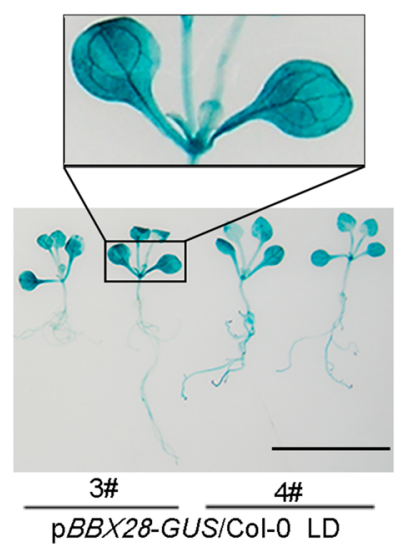

d

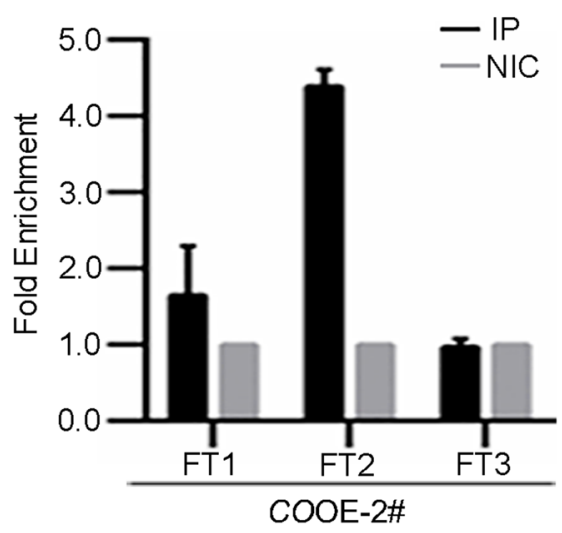

b

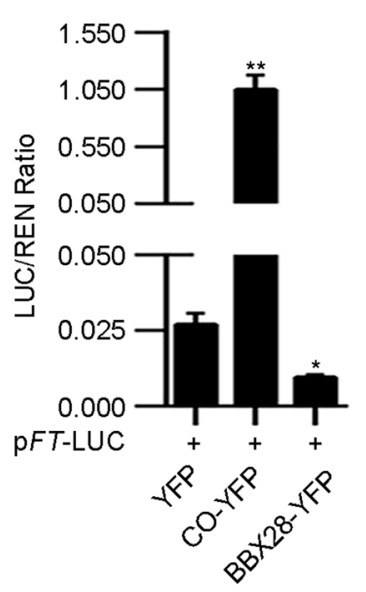

C

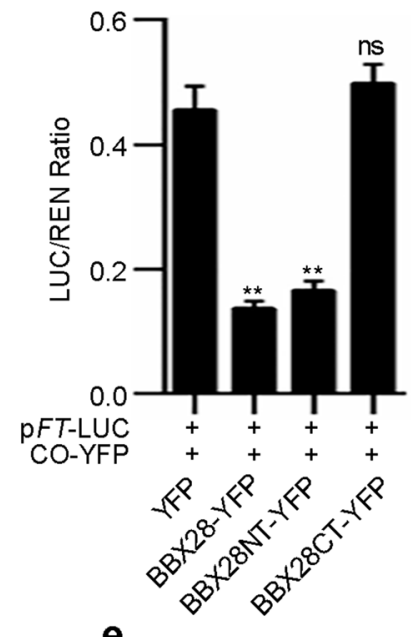

e
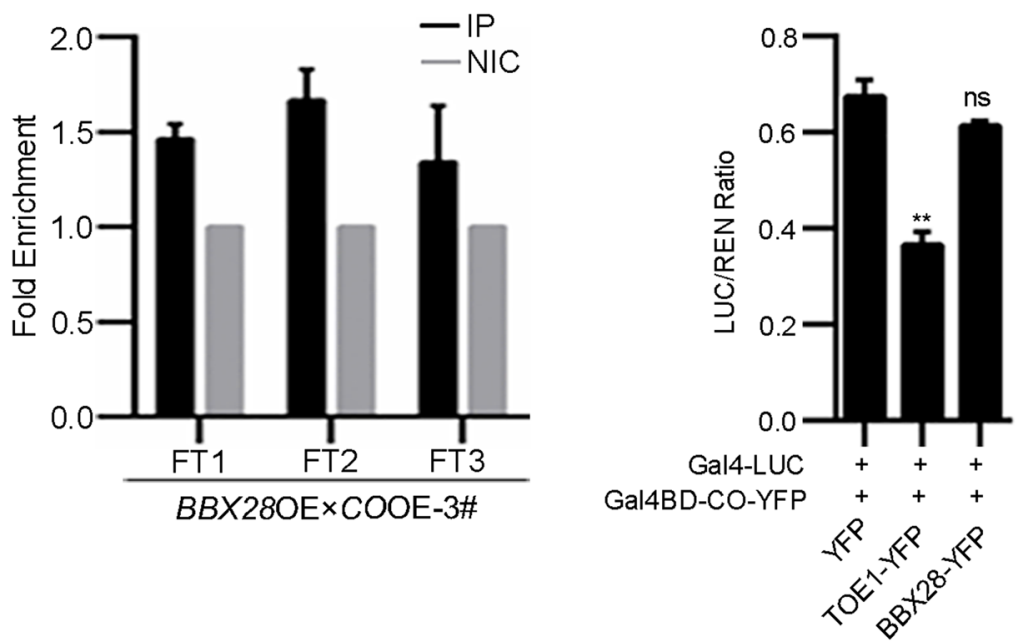

Figure 4. BBX28 inhibits $\mathrm{CO}$ targeting to $F T$ locus without affecting the transcriptional activation activity of CO. (a) Histochemical GUS staining of $\mathrm{p} B B X 28-G U S / \mathrm{Col}-0$ seedlings. Numerals with the pound sign represent independent lines. Bar $=1 \mathrm{~cm}$. (b) Dual-LUC assays show the effects of CO and BBX28 on FT transcription. (c) Dual-LUC assays show the effects of co-expressing CO and BBX28 or BBX28 truncations on FT transcription. (d) ChIP-qPCRs show the effects of BBX28 on CO targeting to FT locus. 12-day-old LD-grown seedlings were harvested at ZT12 for ChIP-qPCR assays using an anti-GFP antibody. IP (GFP antibody): immunoprecipitation; NIC (no GFP antibody): nonimmune control. ChIP results were presented by the fold enrichment. GFP-IP signal relative to corresponding NIC signal was normalized by FT4. Similar results were observed in two independent biological replicates. COOE: 35S-CO-YFP/Col-0; BBX28OE: 35S-BBX28-3×FLAG-mCherry/Col0 . (e) The transcriptional activity assays show the effects of BBX28 on the transcriptional activation activity of CO. YFP served as a negative control. TOE1 served as a positive control. Data are means \pm SEM. In $(\mathbf{b}), n=4$ biological replicates. In (c) and (e), $\mathrm{n}=3$ biological replicates. Statistical significance was analyzed by student's $t$-test; ${ }^{* *} p<0.01,{ }^{*} p<0.05$; ns, not significant.

by $B B X 28 \mathrm{OE} \times \mathrm{COOE}$ (1\# and 5\#) (Fig. S3b), accordantly, the $F T$ transcript level displayed similar patterns to $C O$, with highest in $B B X 28 \mathrm{OE} \times C O O E-3 \#$, followed by $B B X 28 \mathrm{OE} \times C O O E$ (1\# and 5\#) (Fig. 6d). Together, these results suggested that $\mathrm{BBX} 28$ delays flowering in a $\mathrm{CO}$ and $F T$-dependent manner.

\section{Discussion}

The CO-FT module is the major determinant to regulate flowering in response to day length. In this study, we identified a new CO-interacting protein BBX28 which belongs to the Group V B-box transcription factors (BBX26-BBX32). We further dissected a specific role of $\mathrm{N}$-terminal domain of BBX28 in the negative regulation of flowering.

In the light signaling pathways, it was shown that some components function through their different protein domains intelligently. The C-terminal domains of CRY1 and CRY2 (CCT1 and CCT2) interact with COP1 and mediate their signaling responses to light activation. The N-terminal domain of CRY1 (CNT1) mediates its constitutive dimerization, which is required for the light activation of CCT1 activity ${ }^{46,47}$. COP1 is a central switch for light signaling transduction. The nuclear localization signals (NLSs) of COP1 reside in its central core domain while the $\mathrm{N}$-terminal portion acts as the major determinant for its cytoplasmic distribution. The coiled-coil 
a

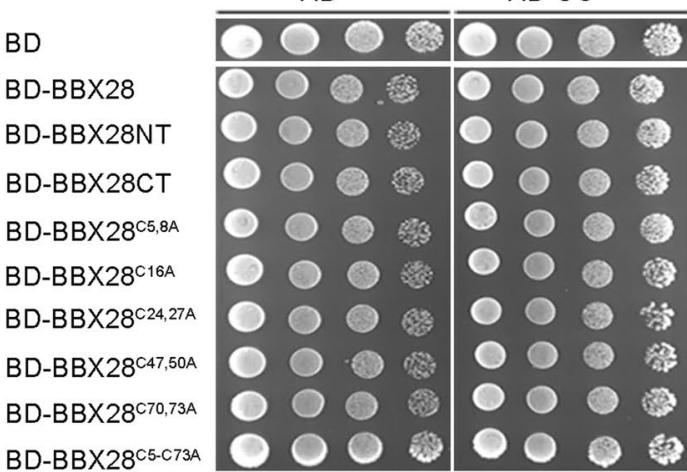

SD-L-T
AD

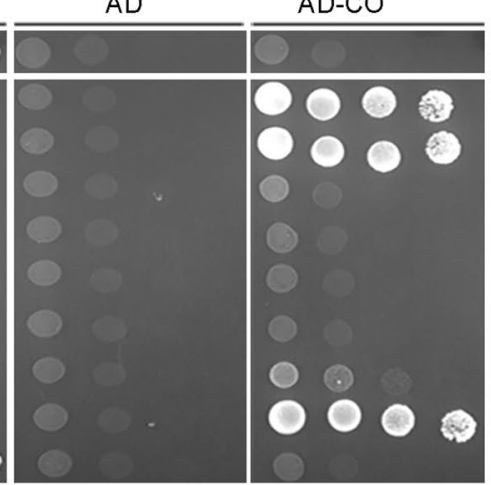

SD-L-T-H-A
AD

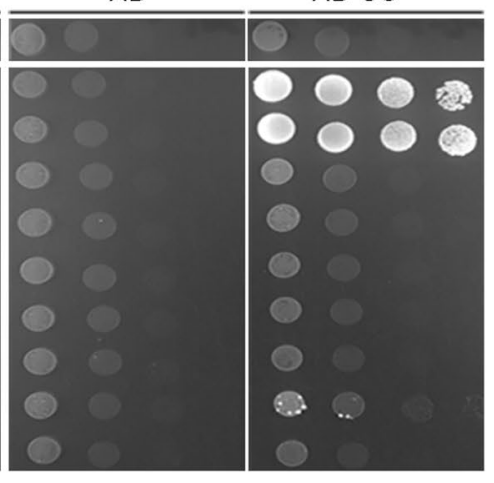

SD-L-T-H+3 mM 3-AT b

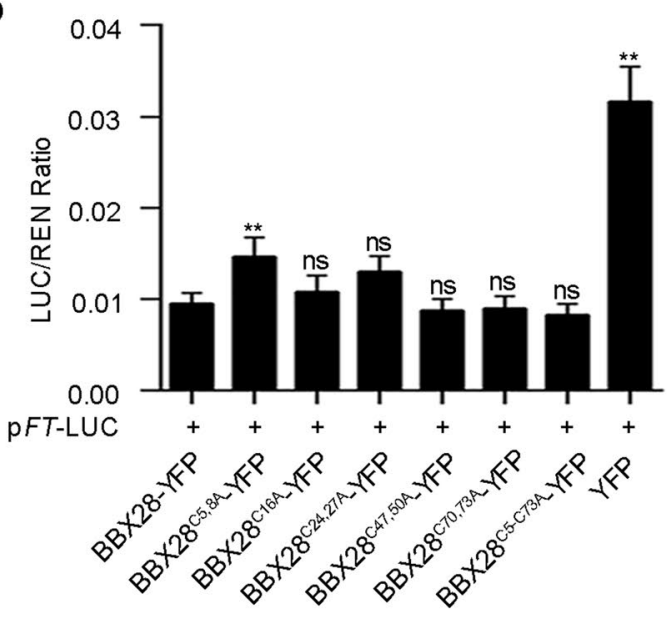

C

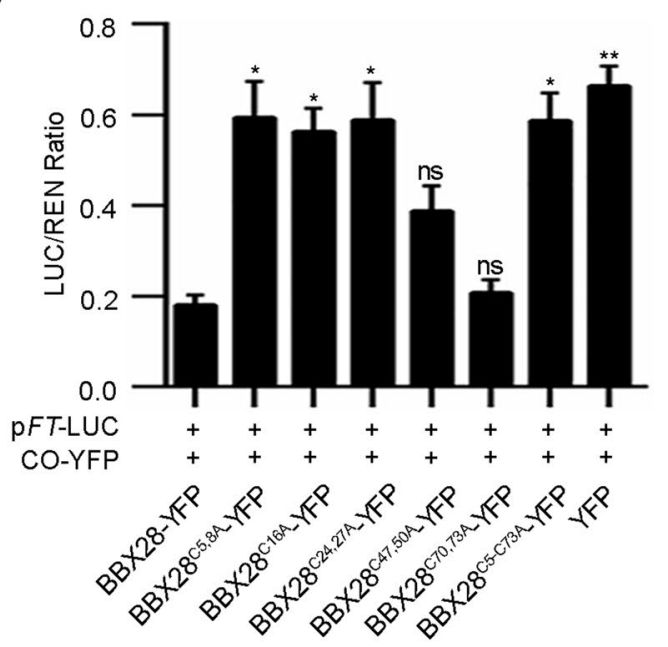

Figure 5. BBX28 N-terminal cysteines mediate heterodimerization between BBX28 and CO and affect the activation of CO on FT transcription. (a) Yeast two-hybrids between CO and BBX28, BBX28 truncations or BBX28 cysteine mutations. Cells were grown on selective plates for interaction assays. (b) Dual-LUC assays show the effects of BBX28 cysteine mutants on FT expression ( $n=4$ biological replicates). (c) Dual-LUC assays show the effects of co-expressing CO and BBX28 cysteine mutants on FT expression ( $\mathrm{n}=3$ biological replicates). Data are means \pm SEM. Statistical significance was analyzed by student's $t$-test; ${ }^{*} p<0.01,{ }^{*} p<0.05$; ns, not significant.

a

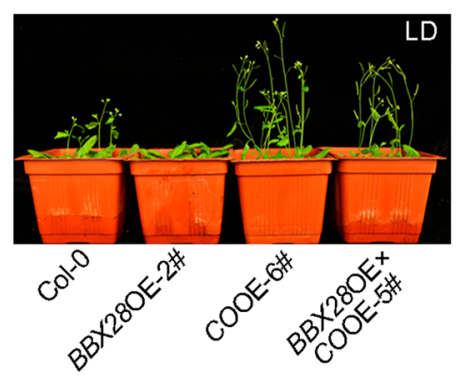

b

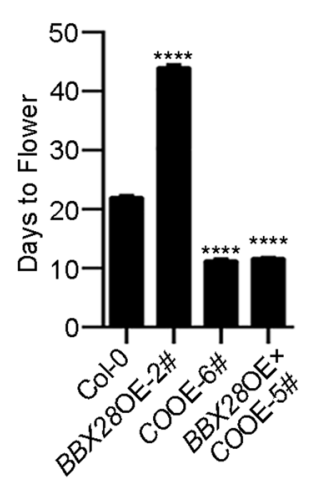

C

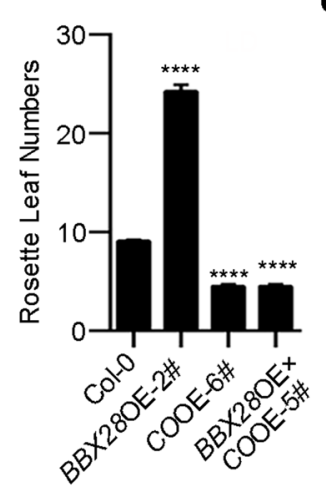

d

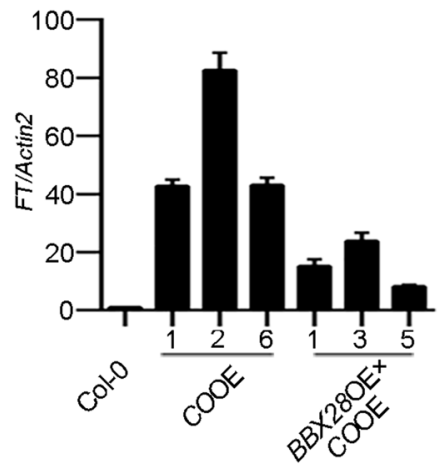

Figure 6. BBX28 genetically regulates flowering through $\mathrm{CO}$ and $F T$. (a) Visual phenotypes of 27-day-old Col-0, $B B X 28, C O$ overexpressing plants or BBX28 and $C O$ co-overexpressing plants under LD. COOE: 35S-COYFP/Col-0; BBX28OE: 35S-BBX28-3 $\times$ FLAG-mCherry/Col-0. $(\mathbf{b}, \mathbf{c})$ Flowering time and rosette leaf numbers of different genotypes in (a). (d) qRT-PCR analyses of $F T$ expression in Col-0, CO overexpressing plants or $B B X 28$ and $C O$ co-overexpressing plants. In $(\mathbf{b}, \mathbf{c})$, data are means \pm SEM $(n=3$ biological replicates; plant numbers $\geq 18$ in each replicate). In (d), Actin 2 was used for data normalization. Data are mean $\pm S D(n=3$ technical replicates). Statistical significance was analyzed by student's $t$-test; ${ }^{* * *} p<0.0001$. 
domain of COP1 mediates its homodimerization and the C-terminal WD40 domain interact with multiple transcription factors or photoreceptors for protein degradation ${ }^{48-50}$. Our results showed that the N-terminus of BBX28 mediates its interaction with $\mathrm{CO}$ and affects the recruitment of $\mathrm{CO}$ to $F T$ locus, resulting in reduction of FT transcription with delayed flowering under LD (Figs. 3, 4 and 6). The late flowering phenotype is relieved when BBX28 N-terminus is deleted (Figs. 1d-f, 4c), supporting specific roles of the regulatory protein domains in the light signaling transduction.

The protein interactions, such as BBX19-CO, BBX32-BBX4 and BBX21-HY5, indicated heterodimer formation among BBXs or between BBX and non-BBX proteins ${ }^{16,27,29,51}$. Arabidopsis BBX32 (AtBBX32) interacted with soybean BBX62 (GmBBX62) through the N-terminal B-box region of BBX3252. Those data suggest the B-box domain plays an essential and conservative role in heterodimerization, but the detailed molecular mechanism for the functioning of BBX domain is still not fully revealed. In this study, the interaction between BBX28 and $\mathrm{CO}(\mathrm{BBX} 1)$ provided another evidence to support heterodimerization of BBX proteins (Fig. 3). In addition, we further showed that several conserved cysteines in the N-terminus of BBX28, particularly those within the B-box domain, are essential for BBX28-CO interaction and the activation of CO on FT transcription (Fig. 5). The structural basis for the role of heterodimerization between BBX28 and CO under light/dark condition in flowering regulation will be of great interest to be investigated in the future.

The evolutionary and structural relationships of BBX proteins have been investigated in many plant species, from algae to monocots and dicots, by the phylogenetic approach ${ }^{53}$. Heading date 1(Hd1), the ortholog of CO in rice, activates flowering under SD while delays flowering time under $\mathrm{LD}^{35}$. OsBBX27 (or OsCO3) and OsBBX5 (or OsCOL4) are negative regulators in plant flowering regulation ${ }^{36,37}$. Overexpressing OsBBX14 or OsCOL9 under both SD and LD delayed the heading date in rice by inhibiting the expressions of florigen genes ${ }^{38,39}$. CmBBX8, a presumed chrysanthemum homolog of Arabidopsis BBX8 (AtBBX8), accelerated flowering by directly targeting CmFLT1, a floral inducer gene ${ }^{40}$. However, CmBBX13 acted as a flowering repressor independently of the photoperiodic pathway ${ }^{41}$. Here we found overexpressing BBX28 delays flowering under LD through CO and FT (Figs. 1 and 6, S5). The delayed flowering of $B B X 28 \mathrm{OE} \times$ COOE plants is comparable to that of COOE plants and the accordant dose curve of $C O$ and FT transcript levels in BBX28 and CO co-overexpressing plants (Figs. 6d, $\mathrm{S} 3 \mathrm{~b})$ supported that BBX28 functions in flowering through CO and FT. Therefore, a new flowering regulator, BBX28, was identified in this study.

BBX28 was known to negatively regulate photomorphogenesis by interacting with ELONGATED HYPOCOTYL 5 (HY5) and interfering with the activity of HY5 in its downstream target gene expression. Besides, COP1 interacts with BBX28 and mediates its protein degradation in darkness via the $26 \mathrm{~S}$ proteasome, demonstrating key roles of BBX28 in COP1-HY5 axis to maintain proper HY5 activity and normal photomorphogenic development ${ }^{42}$. We showed that BBX28 interacts with CO and decreases the recruitment of CO to FT promoter and weakens the activation of CO on FT transcription. The BBX28-HY5 axis in photomorphogenesis and BBX28$\mathrm{CO}-\mathrm{FT}$ axis in flowering regulation indicated that BBX28 plays roles in two different light signaling pathways through diverse interacting proteins to regulate downstream gene expression. It was shown that COP1 interacts with BBX28 and CO and mediates their degradations in darkness ${ }^{9,42}$. The co-localizations of these three proteins in photobodies suggested that BBX28, COP1 and CO may act in the same complex (Fig. S6a). Genetic analyses indicated that the negative role of BBX28 in flowering is CO-dependent under LD (Figs. 6, S5). CO was shown to act genetically downstream of COP1 to regulate flowering 9 . It is therefore of interest to study if and how BBX28 affects COP1-mediated CO degradation in flowering when these three proteins interact with each other. Additionally, we found $C O$ transcription was not affected by BBX28 during the day but decreased about half in $B B X 28 \mathrm{OE}$ plants after dusk (Fig. 2c). It is also of interest to explore the relationship between $B B X 28$ and $C O$ at the transcriptional level.

We proposed a working model for BBX28 in flowering regulation (Fig. S6b). CO binds to FT promoter to activate FT transcription. The interaction between N-terminus of BBX28 and CO decreases the recruitment of CO to FT locus. The N-terminal cysteines play an indispensable role in BBX28-CO heterodimerization and activation of $\mathrm{CO}$ on FT transcription. In wild type (WT/Col-0), the balance between BBX28 and CO maintains precise FT expression, which leads to normal flowering. When BBX28 is over-expressed (BBX28OE), the recruitment of $\mathrm{CO}$ on $F T$ locus is decreased by the overdosed BBX28 protein, resulting in a significant reduction of FT transcript which delays flowering under LD. Considering the similar protein structures of B-box transcription factors, the working model of BBX28 presented here might be applied to other BBX proteins in some degree. In addition, as BBX proteins have roles not only in flowering regulation, but also in photomorphogenesis, shadeavoidance response, abiotic and biotic stresses and plant hormonal pathways ${ }^{27}$, it is therefore of interest to study if and how the working model of BBX28 applies to those signaling pathways.

\section{Methods}

Plant materials and growth conditions. Arabidopsis thaliana ecotype Columbia-0 (Col-0) was used as the wild type for all experiments. The T-DNA insertion mutants of bbx28 (SAIL_828_G11) and co (co-9, SAIL_24_H04) were obtained from the Arabidopsis Biological Resource Center (ABRC), and the homozygous lines were identified by PCR using the primers listed in Table S1. The seeds were surface sterilized and plated on Murashige and Skoog (MS) plates. After stratification for 4 days in dark at $4{ }^{\circ} \mathrm{C}$, the plates were transferred to the growth chambers at $22^{\circ} \mathrm{C}$ with different light conditions based on experimental requirements. The long day (LD) condition was $16 \mathrm{~h}$ light $/ 8 \mathrm{~h}$ dark.

Yeast two-hybrid assays. Yeast two-hybrid interaction assays were performed according to the Yeastmaker Yeast Transformation System 2 User Manual (Clontech). The full lengths of CDS, truncated fragments or mutated sequences of genes of interest were subcloned into pGADT7 or pGBKT7, respectively. The constructs 
were then co-transformed into yeast (AH109) according to the user manual. The yeast cells containing the bait and the prey constructs were grown on selective plates (SD-Leu-Trp, SD-Leu-Trp-His, SD-Leu-Trp-His-Ala and SD-Leu-Trp-His + 3-AT) for analysis. The concentration of 3-AT was $3 \mathrm{mM}$ or $10 \mathrm{mM}$.

Microscopy. Tobacco (N. tabacum) leaves were used for transient expression assays. The constructs were introduced into Agrobacterium tumefaciens strain GV3101 by electroporation and infiltrated into tobacco leaves with an injection syringe. $48 \mathrm{~h}$ later, the infiltrated tobacco leaf disks were subjected to microcopy analyses as described previously. Image stacks of nuclei were subjected to deconvolution by using softWoRx software (Applied Precision) $)^{54-56}$. Co-localization or Nucleolus-tethering System (NoTS) assays were performed as previously described ${ }^{43}$.

Firefly luciferase (LUC) complementation imaging assays. The firefly luciferase (LUC) complementation imaging assay was performed as previously described ${ }^{57}$. BBX28, BBX28NT (1-112 amino acids, aa), BBX28CT (113-223 aa) and CO were fused with the N- or C-terminal fragment of LUC (NLuc and CLuc) respectively. The fused plasmids were introduced into GV3101 by electroporation and then co-infiltrated into tobacco ( $N$. benthamiana) leaves with an injection syringe. $48 \mathrm{~h}$ later, the infiltrated leaves were injected with $100 \mathrm{mM}$ luciferin (Sango, dissolved in water) and the luciferase signals were detected by the PMCapture software (Version 1.00) of a Chemiluminescence Imaging System (Tanon 5500, Shanghai, China).

Co-immunoprecipitation (Co-IP) assays. The plasmid pairs for BBX28-CO interaction assays were coinfiltrated into tobacco ( $N$. benthamiana) leaves. 48 h later, the leaves were collected and ground into good powder in liquid nitrogen for nuclear protein extraction. Samples were re-suspended in $20 \mathrm{ml} \mathrm{CLB1}$ (50 mM HEPES, pH7.5, 150 mM NaCl, 1 mM EDTA, 0.04\% (v/v) $\beta$-mercaptoethanol, $1 \%$ (v/v) Triton X-100, 10\% (v/v) glycerol, $1 \times$ Cocktail) and incubated at $4^{\circ} \mathrm{C}$ for $30 \mathrm{~min}$. The suspensions were filtrated through a double layer of Miracloth (Millipore) twice and centrifuged at $3000 \times g$ for $20 \mathrm{~min}$ at $4{ }^{\circ} \mathrm{C}$. The pellets were washed twice with $1 \mathrm{ml} \mathrm{CLB} 2$ (50 mM HEPES, pH7.5, $150 \mathrm{mM} \mathrm{NaCl}, 1 \mathrm{mM}$ EDTA, 1\% (v/v) Triton X-100, 10\% (v/v) glycerol, $1 \times$ Cocktail) and re-suspended in $200 \mu \mathrm{l} \mathrm{CLB} 2$ added with $20 \mu \mathrm{l} 10 \%(\mathrm{w} / \mathrm{v})$ SDS. The suspensions were sonicated five times and centrifuged at $12,000 \times g$ for $10 \mathrm{~min}$ at $4{ }^{\circ} \mathrm{C}$. Another $1.8 \mathrm{ml} \mathrm{CLB} 2$ buffer was added for resuspension. The nuclear proteins were incubated with $50 \mu \mathrm{l}$ Anti-GFP mAb-Magnetic Beads (MBL) at $4{ }^{\circ} \mathrm{C}$ for $4 \mathrm{~h}$ with rotation. The beads were washed 5 times with washing buffer ( $50 \mathrm{mM}$ HEPES, pH7.5, $150 \mathrm{mM} \mathrm{NaCl}, 10 \%(\mathrm{v} / \mathrm{v})$ glycerol, $0.1 \%(\mathrm{v} / \mathrm{v})$ TritonX-100, $1 \mathrm{mM}$ EDTA, $1 \times$ Cocktail). The proteins were released by boiling at $100^{\circ} \mathrm{C}$ for $5 \mathrm{~min}$ and subjected to Western blotting assays with anti-GFP (Abmart; M20004) and anti-mCherry (Abcam; ab67453) antibodies. Blotting signals were detected by the PMCapture software (Version 1.00) of a Chemiluminescence Imaging System (Tanon 5500, Shanghai, China).

Transgenic plants. The constructs were introduced into GV3101 by electroporation and transformed into Col-0 by the floral-dip method ${ }^{58}$. To obtain transgenic plants in different mutant backgrounds, the plants in Col-0 background were crossed with mutants and the backgrounds were identified by PCRs. Primers used were shown in Table S1.

Site-direct mutagenesis. Site-direct mutagenesis was generated by the two-step overlap PCR. We designed a pair of mutant primers by artificially changing the base sequences of interest. When introducing cysteine to alanine (Cys-to-Ala) substitutions, we changed the bases to GCC. Then PCRs were performed by using the wild type forward primer and mutated reverse primer or the mutated forward primer and wild type reverse primer. The same amounts of the purified PCR products were mixed and used as the template for final PCR, which was performed by using the wild type forward and reverse primers. The site-direct mutagenesis was confirmed by sequencing. Primers used were shown in Table S1.

Flowering time and rosette leaf counting. After surface sterilization and stratification for 4 days in dark at $4{ }^{\circ} \mathrm{C}$, the seeds on plates were transferred to the growth chambers at $22^{\circ} \mathrm{C}$ under $\mathrm{LD}(16 \mathrm{~h} \mathrm{light} / 8 \mathrm{~h}$ dark $)$ for 7 days. Then the seedlings were transferred from the plates to the soil and grown in the greenhouse at $22^{\circ} \mathrm{C}$ under LD. The flowering times and rosette leaf numbers were recorded when plants start bolting.

Quantitative real-time PCR. Expression analyses were performed by quantitative real-time PCR (qRTPCR) as described previously ${ }^{43}$. Total RNAs were extracted from 7-day-old seedlings under the LD condition using the RNeasy Plant Mini Kit (Qiagen). RNAs (about $1 \mu \mathrm{g}$ ) were used as templates for reverse transcription using ReverTra Ace qPCR RT Master Mix with gDNA Remove Kit (Toyobo) according to the manufacturer's instruction. The cDNAs were then diluted and used as templates for qPCR using SYBR Premix Ex Taq (TliRNaseH Plus) in a Bio-Rad CFX96 real-time system. All PCRs were performed by preincubation for 2 min at $95^{\circ} \mathrm{C}$, followed by 50 cycles of denaturation at $95^{\circ} \mathrm{C}$ for $20 \mathrm{~s}$, annealing at $56^{\circ} \mathrm{C}$ for $30 \mathrm{~s}$, and extension at $72{ }^{\circ} \mathrm{C}$ for $30 \mathrm{~s}$. Each reaction was repeated three times. Data were captured and analyzed by Bio-Rad CFX Manager software (Version 2.1) (Bio-Rad). Actin2 (At3g18780) was used for data normalization. Primers used for qRTPCR were listed in Table S1.

Protein extraction and immunoblot assays. For total proteins of plants or seedlings grown under different conditions were extracted by extraction buffer $(50 \mathrm{mM}$ Tris- $\mathrm{HCl}, \mathrm{pH} 7.5,150 \mathrm{mM} \mathrm{NaCl}, 10 \mathrm{mM} \mathrm{MgCl} 2$, $5 \mathrm{mM}$ EDTA, 10\% (v/v) Glycerol, $0.6 \mathrm{mM}$ PMSF, $1 \times$ Cocktail, Roche) $(200 \mu \mathrm{l}$ extraction buffer for about $50 \mu \mathrm{l}$ 
powder). Lysed proteins were placed on ice for $20 \mathrm{~min}$ and mixed several times. Then proteins were centrifuged at $14,000 \times g$ for $10 \mathrm{~min}$ at $4{ }^{\circ} \mathrm{C}$. The supernatants were transformed to new tubes and $5 \times \mathrm{SDS}$-PAGE loading buffer (250mMTris-HCl, pH 6.8, 10\% (w/v) SDS, 0.5\% (w/v) BPB, 50\% (v/v) Glycerol, 5\% (v/v) $\beta$-mercaptoethanol) was added. Samples were boiled at $100{ }^{\circ} \mathrm{C}$ for $5 \mathrm{~min}$ and subjected to SDS-PAGE for Western blotting assays. Blotting signals were detected by the PMCapture software (Version 1.00) of a Chemiluminescence Imaging System (Tanon 5500, Shanghai, China).

Dual-luciferase (dual-LUC) assay. The Dual-LUC assay was performed as described previously ${ }^{59}$. The FT promoter (1800 bp) was subcloned into pGreenII 0800-LUC vector (a kind gift from Prof. Hongtao Liu) as the reporter and full-length of $B B X 28 \mathrm{CDS}, B B X 28$ truncates or point mutations and full-length of CO CDS were subcloned into pCambia131-35S-N1-YFP ${ }^{54}$ as the effectors. pCambia131-35S-YFP (YFP) was used as the control effector. The reporter construct was introduced into the GV3101 harboring pSoup-P19. The effecter constructs were introduced into GV3101. The cells were harvested when grown to OD600 $=0.8-1.0$ and washed once and then resuspended by sterilized water to OD600 $=0.8$. The reporter and the effecter were mixed together at 1:2 ratio for co-infiltration of the tobacco leaves. For assays containing two effectors, the reporter and effectors were mixed together at 1:2:2 ratio. The control and the experimental samples were infiltrated into the same tobacco leaf and more than three independent leaves were served as technical replicates. $48 \mathrm{~h}$ later, the tobacco leaf disks in the infiltrated areas were collected by a puncher with $1.1 \mathrm{~cm}$ in diameter for protein extraction. Extracted proteins were subject to Dual-LUC assays by the Dual-Luciferase Reporter Assay System (Promega E1910) with GloMax 20/20 Luminometer software (version 1.10) (Promega).

The samples were ground in liquid nitrogen in $1.5 \mathrm{ml}$ EP tubes and extracted by $100 \mu \mathrm{l} 1 \times$ Passive Lysis Buffer $(5 \times)$, incubated on ice for $15 \mathrm{~min}$ and mixed several times for efficient lysis. The extracted proteins were centrifuged at $14,000 \times g$ for $10 \mathrm{~min}$ at $4{ }^{\circ} \mathrm{C}$ and supernatants were transformed into new tubes. $8 \mu \mathrm{l}$ supernatant was transformed to a new tube and $40 \mu$ luciferase assay buffer was added and mixed for recording the LUC value. Then $40 \mu \mathrm{l}$ Stop\&Glo buffer was added and mixed for recording the REN value. The LUC/REN ratios were calculated. Data from the luminometer were exported by the GloMax Spreadsheet Interfacer (Promega).

Chromatin immunoprecipitation (ChIP) assay. ChIP assay was performed as previously described ${ }^{60}$. $1 \mathrm{~g}$ 12-day-old seedlings of COOE-2\# (35S-CO-YFP/Col-0) and BBX28OE $\times$ COOE-3\# (35S-BBX28-3 $\times$ FLAG$m$ Cherry/Col- $0 \times 35 S-C O-Y F P / C o l-0)$ were harvested at ZT12 under LD and cross-linked twice in fixing buffer (0.4 M Sucrose, $10 \mathrm{mM}$ Tris-HCl, pH8.0, $1 \mathrm{mM}$ EDTA, 1\% Formaldehyde) for $10 \mathrm{~min}$ by vacuum infiltration. The cross-linking was terminated by the addition of $2.5 \mathrm{M}$ Glycine and vacuum infiltration for $5 \mathrm{~min}$. The samples were ground into good powder, then the nuclei and chromatin were isolated as described in IP assays. $5 \mu \mathrm{g}$ anti-GFP antibody (Abcam) was incubated with $20 \mu \mathrm{l}$ Protein $\mathrm{A}+\mathrm{G}$ magnetic ChIP beads (Millipore) at $4{ }^{\circ} \mathrm{C}$ for 3-5 h following by the addition of extracted chromatins and incubated at $4{ }^{\circ} \mathrm{C}$ overnight. Then the beads were washed by low salt washing buffer (50 mM HEPES, pH7.5, $1 \mathrm{mM}$ EDTA, $150 \mathrm{mM} \mathrm{NaCl}$ ), high salt washing buffer (50 mM HEPES, pH7.5, 1 mM EDTA, $500 \mathrm{mM} \mathrm{NaCl}$ ), LiCl wash buffer (10 mM Tris-HCl, pH8.0, $1 \mathrm{mM}$ EDTA, $0.25 \mathrm{M} \mathrm{LiCl}, 0.5 \%(\mathrm{v} / \mathrm{v}) \mathrm{NP}-40)$ and TE buffer (10 mM Tris-HCl, pH8.0, $1 \mathrm{mM}$ EDTA) and eluted with elution buffer (1\% SDS, $0.1 \mathrm{M} \mathrm{NaHCO}_{3}$ ). After overnight reverse cross-linking, DNA was purified and dissolved in $30 \mu \mathrm{l}$ water. Four fragments (FT1, FT2, FT3 and FT4) of FT locus were used for ChIP qRT-PCR assay ${ }^{17}$. Data were captured and analyzed by Bio-Rad CFX Manager software (Version 2.1) (Bio-Rad). The ChIP results were reported by fold enrichment way using $2^{-\triangle \Delta C t}$ method $^{61}$. GFP-IP signal relative to corresponding nonimmune control (NIC; no GFP antibody) signal was normalized by FT4. Primers used were listed in Table S1.

Transcriptional activity assay. The transcriptional activity assay was performed as previously described with some modifications ${ }^{62}$. The original plasmids were a kind gift from prof. Zhukuan Cheng's lab. The construct $35 S^{\prime}-5 \times$ Gal4-TATA-LUC-Nos was used as the reporter and CaMV35S- $\Omega$-Gal4BD-Nos as the effecter. Gal4BDVP16 was the positive control and pTRL was the internal reference. The $35 S^{\prime}-5 \times$ Gal4-TATA fragment was amplified by the KpnI-35S'-F and KpnI-TATA-R primers using $35 S^{\prime}-5 \times$ Gal4-TATA-LUC-Nos as the template and subcloned into pGreenII 0800-LUC vector to generate pGreenII 0800-35S'-5 × Gal4-TATA-LUC plasmid. The Gal4BD sequence was amplified by BamHI-Gal4BD-F and BamHI-Gal4BD-R primers using CaMV35S$\Omega$-Gal4BD-Nos as the template and subcloned into pCambia131-35S-N1-YFP to generate pCambia131-35SGal4BD-N1-YFP. To construct different effectors, the coding sequences of different genes were subcloned into pCambia131-35S-Gal4BD-N1-YFP plasmid. For the positive control (pCambia131-35S-Gal4BD-VP16-YFP), the VP16 sequence was amplified by SpeI-VP16-F and SpeI-VP16-R primers using Gal4BD-VP16 as the template and subcloned into pCambia131-35S-Gal4BD-N1-YFP. Then constructs of the reporter and effectors were introduced into GV3101 harboring pSoup-P19 or GV3101 and co-infiltrated into tobacco (N. benthamiana) leaves for Dual-LUC assay by the Dual-Luciferase Reporter Assay System (Promega E1910) with GloMax 20/20 Luminometer software (version 1.10) (Promega) to analyze the transcriptional activities. Data from the luminometer were exported by the GloMax Spreadsheet Interface software (Promega).

Histochemical GUS staining. The promoter of BBX28 (2004 bp) was subcloned into pBI101.1-GUS vector and transformed into Col-0 to generate $\mathrm{p} B B X 28-G U S / C o l-0$ transgenic plants. 10-day-old LD grown seedlings were soaked in GUS staining buffer $(1 \mathrm{mg} / \mathrm{ml}$ 5-bromo-4-chloro-3-indolyl-beta-D-glucuronic acid cyclohexylammonium salt (X-Gluc), $50 \mathrm{mM} \mathrm{Na}_{3} \mathrm{PO}_{4}(\mathrm{pH} 7.0), 0.1 \%(\mathrm{v} / \mathrm{v})$ Triton X-100, $2 \mathrm{mM} \mathrm{K}_{4} \mathrm{Fe}(\mathrm{CN})_{6} \cdot 3 \mathrm{H}_{2} \mathrm{O}$, $2 \mathrm{mM} \mathrm{K}_{3} \mathrm{Fe}(\mathrm{CN})_{6}$ and $\left.10 \mathrm{mM} \mathrm{EDTA}\right)$ at $37^{\circ} \mathrm{C}$ overnight in darkness. $75 \%$ ethanol was used to remove chlorophyll for imaging. 
Statistical analyses. For quantitative real-time PCR, flowering time and rosette leaf counting, Dual-LUC assay and transcriptional activity assay, the differences between samples were analyzed by student's t-test. For all analyses, statistical significance was set as ${ }^{* * *} p<0.0001,{ }^{* *} p<0.001,{ }^{* *} p<0.01,{ }^{*} p<0.05$, and ns (not significant).

\section{Data availability}

All necessary data generated or analyzed during the present study are included in this published article and its Supplementary Information files.

Received: 21 April 2020; Accepted: 1 October 2020

Published online: 20 October 2020

\section{References}

1. Srikanth, A. \& Schmid, M. Regulation of flowering time: all roads lead to Rome. Cell. Mol. Life Sci. 68, 2013-2037 (2011).

2. Fornara, F., de Montaigu, A. \& Coupland, G. SnapShot: control of flowering in Arabidopsis. Cell 141, 550 (2010) ((e551-552)).

3. Zhang, H. et al. The effects of Arabidopsis genome duplication on the chromatin organization and transcriptional regulation. Nucleic Acids Res. 47, 7857-7869 (2019).

4. Shim, J. S., Kubota, A. \& Imaizumi, T. Circadian clock and photoperiodic flowering in Arabidopsis: CONSTANS is a hub for signal integration. Plant Physiol. 173, 5-15 (2017).

5. Tiwari, S. B. et al. The flowering time regulator CONSTANS is recruited to the FLOWERING LOCUS T promoter via a unique cis-element. New Phytol. 187, 57-66 (2010).

6. Abe, M. et al. FD, a bZIP protein mediating signals from the floral pathway integrator FT at the shoot apex. Science 309, $1052-1056$ (2005).

7. Shim, J. S. \& Imaizumi, T. Circadian clock and photoperiodic response in Arabidopsis: from seasonal flowering to redox homeostasis. Biochemistry 54, 157-170 (2015).

8. Lazaro, A., Valverde, F., Pineiro, M. \& Jarillo, J. A. The Arabidopsis E3 ubiquitin ligase HOS1 negatively regulates CONSTANS abundance in the photoperiodic control of flowering. Plant Cell 24, 982-999 (2012).

9. Liu, L. J. et al. COP1-mediated ubiquitination of CONSTANS is implicated in cryptochrome regulation of flowering in Arabidopsis. Plant Cell 20, 292-306 (2008).

10. Lazaro, A., Mouriz, A., Pineiro, M. \& Jarillo, J. A. Red light-mediated degradation of CONSTANS by the E3 ubiquitin ligase HOS1 regulates photoperiodic flowering in Arabidopsis. Plant Cell 27, 2437-2454 (2015).

11. Song, Y. H. et al. Distinct roles of FKF1, Gigantea, and zeitlupe proteins in the regulation of constans stability in Arabidopsis photoperiodic flowering. Proc. Natl. Acad. Sci. USA 111, 17672-17677 (2014).

12. Valverde, F. et al. Photoreceptor regulation of CONSTANS protein in photoperiodic flowering. Science 303, 1003-1006 (2004).

13. Zuo, Z., Liu, H., Liu, B., Liu, X. \& Lin, C. Blue light-dependent interaction of CRY2 with SPA1 regulates COP1 activity and floral initiation in Arabidopsis. Curr. Biol. 21, 841-847 (2011).

14. Cheng, Z. et al. Nup96 and HOS1 are mutually stabilized and gate CONSTANS protein level, conferring long-day photoperiodic flowering regulation in Arabidopsis. Plant Cell 32, 374-391 (2019).

15. Hwang, D. Y. et al. GIGANTEA regulates the timing stabilization of CONSTANS by altering the interaction between FKF1 and ZEITLUPE. Mol. Cells 42, 693-701 (2019).

16. Wang, C. Q., Guthrie, C., Sarmast, M. K. \& Dehesh, K. BBX19 interacts with CONSTANS to repress FLOWERING LOCUS T transcription, defining a flowering time checkpoint in Arabidopsis. Plant Cell 26, 3589-3602 (2014).

17. Nguyen, K. T., Park, J., Park, E., Lee, I. \& Choi, G. The Arabidopsis RING domain protein BOI inhibits flowering via CO-dependent and CO-independent mechanisms. Mol. Plant 8, 1725-1736 (2015).

18. Zhang, B., Wang, L., Zeng, L., Zhang, C. \& Ma, H. Arabidopsis TOE proteins convey a photoperiodic signal to antagonize CONSTANS and regulate flowering time. Genes Dev. 29, 975-987 (2015).

19. Graeff, M. et al. Microprotein-mediated recruitment of CONSTANS into a TOPLESS trimeric complex represses flowering in Arabidopsis. PLoS Genet. 12, e1005959 (2016).

20. Xu, F. et al. DELLA proteins physically interact with CONSTANS to regulate flowering under long days in Arabidopsis. FEBS Lett. 590, 541-549 (2016).

21. Cao, S. et al. A distal CCAAT/NUCLEAR FACTOR Y complex promotes chromatin looping at the FLOWERING LOCUS T promoter and regulates the timing of flowering in Arabidopsis. Plant Cell 26, 1009-1017 (2014).

22. Kumar, S., Choudhary, P., Gupta, M. \& Nath, U. VASCULAR PLANT ONE-ZINC FINGER1 (VOZ1) and VOZ2 interact with CONSTANS and promote photoperiodic flowering transition. Plant Physiol. 176, 2917-2930 (2018).

23. Serrano-Bueno, G. et al. CONSTANS-FKBP12 interaction contributes to modulation of photoperiodic flowering in Arabidopsis. Plant J. 101, 1287-1302 (2019).

24. Hayama, R. et al. PSEUDO RESPONSE REGULATORs stabilize CONSTANS protein to promote flowering in response to day length. EMBO J. 36, 904-918 (2017).

25. Liu, Y. et al. CIB1 and CO interact to mediate CRY2-dependent regulation of flowering. EMBO Rep. 19, 10 (2018).

26. Zhang, R., Yang, C., Jiang, Y. \& Li, L. A PIF7-CONSTANS-centered molecular regulatory network underlying shade-accelerated flowering. Mol. Plant 12, 1587-1597 (2019).

27. Gangappa, S. N. \& Botto, J. F. The BBX family of plant transcription factors. Trends Plant Sci. 19, 460-470 (2014).

28. Song, Z., Bian, Y., Liu, J., Sun, Y. \& Xu, D. B-box proteins: pivotal players in light-mediated development in plants. J. Integr. Plant Biol. 62, 1293-1302 (2020).

29. Tripathi, P., Carvallo, M., Hamilton, E. E., Preuss, S. \& Kay, S. A. Arabidopsis B-BOX32 interacts with CONSTANS-LIKE3 to regulate flowering. Proc. Natl. Acad. Sci. USA 114, 172-177 (2017).

30. Datta, S., Hettiarachchi, G. H., Deng, X. W. \& Holm, M. Arabidopsis CONSTANS-LIKE3 is a positive regulator of red light signaling and root growth. Plant Cell 18, 70-84 (2006).

31. Hassidim, M., Harir, Y., Yakir, E., Kron, I. \& Green, R. M. Over-expression of CONSTANS-LIKE 5 can induce flowering in shortday grown Arabidopsis. Planta 230, 481-491 (2009).

32. Cheng, X. F. \& Wang, Z. Y. Overexpression of COL9, a CONSTANS-LIKE gene, delays flowering by reducing expression of CO and FT in Arabidopsis thaliana. Plant J. 43, 758-768 (2005).

33. Li, F. et al. The B-box family gene STO (BBX24) in Arabidopsis thaliana regulates flowering time in different pathways. PLoS ONE 9, e87544 (2014).

34. Park, H. Y. et al. EMF1 interacts with EIP1, EIP6 or EIP9 involved in the regulation of flowering time in Arabidopsis. Plant Cell Physiol. 52, 1376-1388 (2011).

35. Yano, M. et al. Hd1, a major photoperiod sensitivity quantitative trait locus in rice, is closely related to the Arabidopsis flowering time gene CONSTANS. Plant Cell 12, 2473-2484 (2000). 
36. Kim, S. K. et al. OsCO3, a CONSTANS-LIKE gene, controls flowering by negatively regulating the expression of FT-like genes under SD conditions in rice. Planta 228, 355-365 (2008).

37. Lee, Y. S. et al. OsCOL4 is a constitutive flowering repressor upstream of Ehd1 and downstream of OsphyB. Plant J. 63, 18-30 (2010).

38. Bai, B. et al. OsBBX14 delays heading date by repressing florigen gene expression under long and short-day conditions in rice. Plant Sci. 247, 25-34 (2016).

39. Liu, H. et al. CONSTANS-like 9 (COL9) delays the flowering time in Oryza sativa by repressing the Ehd1 pathway. Biochem. Biophys. Res. Commun. 479, 173-178 (2016).

40. Wang, L. et al. CmBBX8 accelerating flowering by targeting CmFTL1 directly in summer chrysanthemum. Plant Biotechnol. J 18, 1562-1572 (2019).

41. Ping, Q. et al. The heterologous expression in Arabidopsis thaliana of a chrysanthemum gene encoding the BBX family transcription factor CmBBX13 delays flowering. Plant Physiol. Biochem. 144, 480-487 (2019).

42. Lin, F. et al. B-BOX DOMAIN PROTEIN28 negatively regulates photomorphogenesis by repressing the activity of transcription factor HY5 and undergoes COP1-mediated degradation. Plant Cell 30, 2006-2019 (2018).

43. Liu, Y., Liu, Q., Yan, Q., Shi, L. \& Fang, Y. Nucleolus-tethering system (NoTS) reveals that assembly of photobodies follows a selforganization model. Mol. Biol. Cell 25, 1366-1373 (2014).

44. An, H. et al. CONSTANS acts in the phloem to regulate a systemic signal that induces photoperiodic flowering of Arabidopsis. Development 131, 3615-3626 (2004).

45. Kumar, S. V. et al. Transcription factor PIF4 controls the thermosensory activation of flowering. Nature 484(7393), 242-245 (2012).

46. Sang, Y. et al. N-terminal domain-mediated homodimerization is required for photoreceptor activity of Arabidopsis CRYPTOCHROME 1. Plant Cell 17, 1569-1584 (2005).

47. Yang, H. Q. et al. The C termini of Arabidopsis cryptochromes mediate a constitutive light response. Cell 103, 815-827 (2000).

48. Holm, M. \& Deng, X. W. Structural organization and interactions of COP1, a light-regulated developmental switch. Plant Mol. Biol. 41, 151-158 (1999).

49. Stacey, M. G., Hicks, S. N. \& von Arnim, A. G. Discrete domains mediate the light-responsive nuclear and cytoplasmic localization of Arabidopsis COP1. Plant Cell 11, 349-364 (1999).

50. Lau, O. S. \& Deng, X. W. The photomorphogenic repressors COP1 and DET1: 20 years later. Trends Plant. Sci. 17, 584-593 (2012).

51. Holtan, H. E. et al. BBX32, an Arabidopsis B-Box protein, functions in light signaling by suppressing HY5-regulated gene expression and interacting with STH2/BBX21. Plant Physiol. 156, 2109-2123 (2011).

52. Qi, Q. et al. Involvement of the N-terminal B-box domain of Arabidopsis BBX32 protein in interaction with soybean BBX62 protein. J. Biol. Chem. 287, 31482-31493 (2012).

53. Crocco, C. D. \& Botto, J. F. BBX proteins in green plants: insights into their evolution, structure, feature and functional diversification. Gene 531, 44-52 (2013).

54. Fang, Y. \& Spector, D. L. Identification of nuclear dicing bodies containing proteins for microRNA biogenesis in living Arabidopsis plants. Curr. Biol. 17, 818-823 (2007).

55. Fang, Y. \& Spector, D. L. Live cell imaging of plants. Cold Spring Harb. Protoc. 2010, 68 (2010).

56. Shi, L., Wang, J., Hong, F., Spector, D. L. \& Fang, Y. Four amino acids guide the assembly or disassembly of Arabidopsis histone H33-containing nucleosomes. Proc Natl Acad Sci USA 108, 10574-10578 (2011).

57. Chen, H. et al. Firefly luciferase complementation imaging assay for protein-protein interactions in plants. Plant Physiol. 146, 368-376 (2008).

58. Clough, S. J. \& Bent, A. F. Floral dip: a simplified method for agrobacterium-mediated transformation of Arabidopsis thaliana. Plant J. 16, 735-743 (1998).

59. Liu, H. et al. Photoexcited CRY2 interacts with CIB1 to regulate transcription and floral initiation in Arabidopsis. Science 322, 1535-1539 (2008).

60. Saleh, A., Alvarez-Venegas, R. \& Avramova, Z. An efficient chromatin immunoprecipitation (ChIP) protocol for studying histone modifications in Arabidopsis plants. Nat. Protoc. 3, 1018-1025 (2008).

61. Gnesutta, N. et al. CONSTANS imparts DNA sequence specificity to the histone fold NF-YB/NF-YC dimer. Plant Cell 29, 15161532 (2017).

62. Wu, X., Tang, D., Li, M., Wang, K. \& Cheng, Z. Loose Plant Architecture1, an INDETERMINATE DOMAIN protein involved in shoot gravitropism, regulates plant architecture in rice. Plant Physiol. 161, 317-329 (2013).

\section{Acknowledgements}

We gratefully acknowledge the Arabidopsis Biological Resource Center for sending seeds of lines used in this study. We thank Prof. Hongtao Liu and Prof. Zhukuan Cheng for the kind plasmids. We also thank Prof. Fang's lab members for suggestions on the manuscript writing. This work was supported by National Science Foundation of China (31871230 to Y.F.).

\section{Author contributions}

Y.L. and Y.F. designed the experiments. Y.L. performed most of experiments, G.L., and C.Y. assisted in experiments. Y.L., C.Y., G.L. and Y.F. wrote the manuscript. All authors reviewed the manuscript.

\section{Competing interests}

The authors declare no competing interests.

\section{Additional information}

Supplementary information is available for this paper at https://doi.org/10.1038/s41598-020-74445-7.

Correspondence and requests for materials should be addressed to Y.F.

Reprints and permissions information is available at www.nature.com/reprints.

Publisher's note Springer Nature remains neutral with regard to jurisdictional claims in published maps and institutional affiliations. 
(c) (i) Open Access This article is licensed under a Creative Commons Attribution 4.0 International cc) License, which permits use, sharing, adaptation, distribution and reproduction in any medium or format, as long as you give appropriate credit to the original author(s) and the source, provide a link to the Creative Commons licence, and indicate if changes were made. The images or other third party material in this article are included in the article's Creative Commons licence, unless indicated otherwise in a credit line to the material. If material is not included in the article's Creative Commons licence and your intended use is not permitted by statutory regulation or exceeds the permitted use, you will need to obtain permission directly from the copyright holder. To view a copy of this licence, visit http://creativecommons.org/licenses/by/4.0/.

(C) The Author(s) 2020 